\title{
DIVIDED DIFFERENCES AND THE WEYL CHARACTER FORMULA IN EQUIVARIANT K-THEORY
}

\author{
Megumi Harada, Gregory D. Landweber, and Reyer Sjamaar
}

AbStract. Let $X$ be a topological space and $G$ a compact connected Lie group acting on $X$. Atiyah proved that the $G$-equivariant $\mathrm{K}$-group of $X$ is a direct summand of the $T$-equivariant K-group of $X$, where $T$ is a maximal torus of $G$. We show that this direct summand is equal to the subgroup of $K_{T}^{*}(X)$ annihilated by certain divided difference operators. If $X$ consists of a single point, this assertion amounts to the Weyl character formula. We also give sufficient conditions on $X$ for $K_{G}^{*}(X)$ to be isomorphic to the subgroup of Weyl invariants of $K_{T}^{*}(X)$.

\section{Introduction}

Hermann Weyl's theorem of the maximal torus allows one for many purposes to pass from a compact connected Lie group $G$ to a maximal torus $T$. A famous example of this principle is the Weyl character formula, which enables one to compute the characters of the irreducible representations of $G$ in terms of the characters of $T$ and the action of the Weyl group on $T$.

One of the results of this paper is an extension of the Weyl character formula to the equivariant K-theory of a compact $G$-space $X$. Atiyah [2] proved that the restriction map from the $G$-equivariant K-ring $K_{G}^{*}(X)$ to the $T$-equivariant K-ring $K_{T}^{*}(X)$ has a natural left inverse. This "wrong-way" or pushforward homomorphism is defined by means of the Dolbeault operator associated with an invariant complex structure on the homogeneous space $G / T$. Although Atiyah proved that $K_{G}^{*}(X)$ is a direct summand of $K_{T}^{*}(X)$, he did not tell whether this direct summand is determined by the Weyl group action on $K_{T}^{*}(X)$. In fact, it is easy to see that $K_{G}^{*}(X)$ is contained in the Weyl invariants of $K_{T}^{*}(X)$, but an example of McLeod [19] (see also Example 4.8) shows that this inclusion is in general not an equality.

In this paper we show that the action of the Weyl group $W$ on $K_{T}^{*}(X)$ extends to an action of a Hecke ring $\mathcal{D}$ generated by divided difference operators, which was introduced in the context of Schubert calculus by Demazure [9, 10]. This $\mathcal{D}$-action is analogous to a $\mathcal{D}$-action on the K-theory of the classifying space $B T$ previously defined by Bressler and Evens [7]. The ring $\mathcal{D}$ contains a left ideal $I(\mathcal{D})$, which we dub the augmentation left ideal by analogy with the augmentation ideal of the group ring of $W$. Our main result, Theorem 4.6, is that $K_{G}^{*}(X)$ is isomorphic to the subring of $K_{T}^{*}(X)$ annihilated by $I(\mathcal{D})$. In other words, a $T$-equivariant class is $G$-equivariant if and only if it is killed by the divided difference operators. This result is an application of the work of Demazure and of various structural theorems regarding the rings $R(T)$ and $\mathcal{D}$ due to Pittie [23], Steinberg [31], Kazhdan and Lusztig [15], and Kostant and Kumar [17].

Received by the editors June 9,2009

2000 Mathematics Subject Classification. 19E08, 19L47. 
It turns out (Theorem 4.7) that the divided difference operator associated with the longest Weyl group element acts on $K_{T}^{*}(X)$ as a projection onto the direct summand $K_{G}^{*}(X)$. For a space $X$ consisting of a single point, this statement is equivalent to the Weyl character formula. Thus our results can be viewed as "lifting" the Weyl character formula from a point to an arbitrary $G$-space.

There is a natural condition under which $K_{G}^{*}(X)$ is isomorphic to the Weyl invariant part of $K_{T}^{*}(X)$, namely that the Weyl denominator, viewed as an element of the representation ring $R(T)$, should not be a zero divisor in $K_{T}^{*}(X)$ (Theorem 4.9). This condition is satisfied for interesting classes of spaces, such as nonsingular projective varieties on which $G$ acts linearly (Corollary 4.10).

A by-product of our work is a form of duality. There is a natural bilinear pairing on the $K_{G}^{*}(X)$-module $K_{T}^{*}(X)$ defined by pushing forward to $K_{G}^{*}(X)$ the product of two classes in $K_{T}^{*}(X)$. Proposition 5.1 states that $K_{T}^{*}(X)$ is isomorphic to its dual via this "intersection" pairing.

All these results generalize when we replace the maximal torus $T$ by a closed connected subgroup of $G$ which contains $T$. In this manner we obtain a lift of the GrossKostant-Ramond-Sternberg character formula to a topological $G$-space. We hope to take this up in a sequel to this paper.

The treatment of these problems in equivariant K-theory is parallel to the case of Borel's equivariant cohomology theory $H_{G}^{*}(X)$, which was covered in [13]. (For instance, for reasonable coefficient rings we have $H_{G}^{*}(X) \cong H_{T}^{*}(X)^{W}$ provided that the discriminant of $G$ is not a zero divisor in $H_{T}^{*}(X)$. Thus the Weyl denominator plays a rôle in K-theory analogous to that of the discriminant in cohomology.) However, there are several significant differences between the two theories as well, and for this reason we have chosen to provide full details. One such difference is the fact that the restriction map $H_{G}^{*}(X) \rightarrow H_{T}^{*}(X)$ becomes injective only after inverting the torsion primes of $G$. In K-theory these primes cause no trouble.

Our results have counterparts in the equivariant K-theory of algebraic varieties, as follows from work of Merkurjev [20], and in the equivariant K-theory and KK-theory of nuclear $C^{*}$-algebras, as follows from work of Rosenberg and Schochet [26]. We present the case of algebraic K-theory in Section 6.

\section{Divided differences}

Notation. Throughout this paper, with the exception of Section $6, G$ denotes a compact connected Lie group, $\mathcal{X}(G)=\operatorname{Hom}(G, \mathbf{U}(1))$ the character group of $G$, and $R(G)$ the Grothendieck ring of finite-dimensional complex $G$-modules. We choose once and for all a maximal torus $T$ of $G$. Let $j: T \rightarrow G$ be the inclusion map and $W=N_{G}(T) / T$ the Weyl group. Then $R(T)$ is canonically isomorphic to the group ring $\mathbf{Z}[\mathcal{X}(T)]$ and the restriction homomorphism $j^{*}: R(G) \rightarrow R(T)$ induces an isomorphism $R(G) \cong R(T)^{W}$. (See e.g. [6, $\S$ IX.3].) Let $\mathcal{R} \subseteq \mathcal{X}(T)$ be the root system of $(G, T)$. As in $\left[6, \S\right.$ VI.3], we denote by $e^{\lambda}$ the element of $R(T)$ defined by a character $\lambda \in \mathcal{X}(T)$. We fix a basis of the root system $\mathcal{R}$ and we let

$$
\rho=\frac{1}{2} \sum_{\alpha \in \mathcal{R}^{+}} \alpha \in \frac{1}{2} \mathcal{X}(T)
$$

be the half-sum of the positive roots. 
Holomorphic induction. See [2, $\S 4],[3, \S I I .5],[5]$ and [27] for the material in this subsection. Let $G_{\mathbf{C}}$ be the complexification of $G$ and let $B^{-}$be the Borel subgroup of $G_{\mathbf{C}}$ with Lie algebra

$$
\mathfrak{b}^{-}=\mathfrak{t}_{\mathbf{C}} \oplus \bigoplus_{\alpha \in \mathcal{R}^{+}} \mathfrak{g}_{\mathbf{C}}^{-\alpha}
$$

where $\mathfrak{g}_{\mathbf{C}}^{\alpha}$ denotes the root space of the root $\alpha$. The inclusion $G \rightarrow G_{\mathbf{C}}$ descends to a $G$-equivariant diffeomorphism $G / T \cong G_{\mathbf{C}} / B^{-}$.

Let $V$ be a finite-dimensional complex $T$-module. Extend $V$ to a $B^{-}$-module by letting the nilradical of $B^{-}$act trivially. Let $\left(\Lambda^{0, *} E_{V}, \bar{\partial}_{V}\right)$ be the Dolbeault complex on $G_{\mathbf{C}} / B^{-}$with coefficients in the homogeneous holomorphic vector bundle $E_{V}=$ $G_{\mathbf{C}} \times{ }^{B^{-}} V$. The equivariant index of $\bar{\partial}_{V}$ is a virtual $G$-module and depends only on the class of $V$ in $R(T)$. The map $j_{*}: R(T) \rightarrow R(G)$ defined by $[V] \mapsto \operatorname{index}\left(\bar{\partial}_{V}\right)$ is the pushforward homomorphism or holomorphic induction map. It satisfies $j_{*}(1)=1$ and is $R(G)$-linear,

$$
j_{*}\left(j^{*}(v) u\right)=v j_{*}(u)
$$

for all $u \in R(T)$ and $v \in R(G)$. It follows that $j_{*} j^{*}(v)=v$ for all $v \in R(G)$. The Lefschetz fixed point formula gives an expression for the $R(G)$-linear endomorphism $j^{*} j_{*}$ of $R(T)$, namely

$$
j^{*} j_{*}(u)=\frac{J(u)}{\mathbf{d}}
$$

for all $u \in R(T)$. Here $J$ is the $\mathbf{Z}$-linear endomorphism of $R(T)$ defined by

$$
J(u)=\sum_{w \in W} \operatorname{det}(w) w \odot u,
$$

where $w \odot u=e^{-\rho} w\left(e^{\rho} u\right)$ is the $\rho$-shifted $W$-action on $R(T)$, which is well-defined because $\rho-w(\rho) \in \mathcal{X}(T)$ for all $w \in W$. The denominator is

$$
\mathbf{d}=J(1)=\sum_{w \in W} \operatorname{det}(w) w \odot 1=\prod_{\alpha \in \mathcal{R}^{+}}\left(1-e^{-\alpha}\right) .
$$

By the Borel-Weil theorem, $j_{*}\left(e^{\lambda}\right)=\left[M_{\lambda}\right]$ for all dominant $\lambda \in \mathcal{X}(T)$, where $M_{\lambda}$ is the irreducible $G$-module with highest weight $\lambda$. Therefore (1.2) is equivalent to the Weyl character formula.

Demazure's operators. Demazure $[9,10]$ noticed that the endomorphism $j^{*} j_{*}$ of $R(T)$ can be factorized into a composition of $N$ operators, where $N=\left|\mathcal{R}^{+}\right|$. These operators are defined as follows.

Let $\alpha$ be a root and let $s_{\alpha} \in W$ be the reflection in $\alpha$. For every $\lambda \in \mathcal{X}(T)$, the element $e^{\lambda}-e^{-\alpha} e^{s_{\alpha}(\lambda)}$ of $R(T)$ is uniquely divisible by $1-e^{-\alpha}$, so we have a $\mathbf{Z}$-linear endomorphism $\delta_{\alpha}$ of $R(T)$ defined by

$$
\delta_{\alpha}(u)=\frac{u-e^{-\alpha} s_{\alpha}(u)}{1-e^{-\alpha}} .
$$

This map, known in the recent literature as an isobaric divided difference operator, was introduced by Demazure in [10] and denoted by $\Lambda_{\alpha}^{0}$ there. He considered only the 
simply connected case, but $\delta_{\alpha}$ is well-defined for any $G$. The following properties are easily deduced from the definition:

$$
\begin{gathered}
\delta_{\alpha}(1)=1, \quad \delta_{\alpha}^{2}=\delta_{\alpha}, \\
s_{\alpha} \delta_{\alpha}=\delta_{\alpha}, \quad \delta_{\alpha} s_{\alpha}=\delta_{-\alpha}=1+e^{\alpha}-e^{\alpha} \delta_{\alpha}, \quad w \delta_{\alpha} w^{-1}=\delta_{w(\alpha)}, \\
\delta_{\alpha}\left(u_{1} u_{2}\right)=\delta_{\alpha}\left(u_{1}\right) u_{2}+s_{\alpha}\left(u_{1}\right)\left(\delta_{\alpha}\left(u_{2}\right)-u_{2}\right),
\end{gathered}
$$

for all $\alpha \in \mathcal{R}, w \in W$ and $u_{1}, u_{2} \in R(T)$. In addition, Demazure defined the operators (cf. also his earlier papers $[8,9]$ )

$$
\delta_{\alpha}^{\prime}(u)=\frac{u-s_{\alpha}(u)}{1-e^{-\alpha}} .
$$

It is plain that

$$
\delta_{\alpha}^{\prime}(1)=0, \quad\left(\delta_{\alpha}^{\prime}\right)^{2}=\delta_{\alpha}^{\prime}, \quad \delta_{\alpha}^{\prime}=e^{\alpha}\left(\delta_{\alpha}-1\right),
$$

where in the first equality 1 denotes the identity element of $R(T)$ and in the third the identity automorphism of $R(T)$. The product rule is

$$
\begin{gathered}
\delta_{\alpha}^{\prime}\left(u_{1} u_{2}\right)=\delta_{\alpha}^{\prime}\left(u_{1}\right) u_{2}+s_{\alpha}\left(u_{1}\right) \delta_{\alpha}^{\prime}\left(u_{2}\right), \\
\delta_{\alpha}\left(u_{1} u_{2}\right)=\delta_{\alpha}\left(u_{1}\right) u_{2}+e^{-\alpha} s_{\alpha}\left(u_{1}\right) \delta_{\alpha}^{\prime}\left(u_{2}\right),
\end{gathered}
$$

where the second line is just an alternative form of (1.8).

Demazure proved that the $\delta_{\alpha}$ satisfy braid relations and deduced the following statement. (His proof has an error, which was corrected by Andersen [1] and Ramanan and Ramanathan [25].)

1.1. Theorem ([10, Théorème 1]). For every $w \in W$ and for every reduced expression $w=s_{\beta_{1}} s_{\beta_{2}} \cdots s_{\beta_{l}}$ in terms of simple reflections, the composition $\delta_{\beta_{1}} \delta_{\beta_{2}} \cdots \delta_{\beta_{l}}$ takes the same value $\partial_{w}$; and the composition $\delta_{\beta_{1}}^{\prime} \delta_{\beta_{2}}^{\prime} \cdots \delta_{\beta_{l}}^{\prime}$ takes the same value $\partial_{w}^{\prime}=e^{\rho} \partial_{w} e^{-\rho}$.

(A comment on the last identity: if $\rho \notin \mathcal{X}(T)$, then multiplication by $e^{ \pm \rho}$ does not preserve $\mathbf{Z}[\mathcal{X}(T)]$, but the operator $e^{\rho} \partial_{w} e^{-\rho}$ does preserve $\mathbf{Z}[\mathcal{X}(T)]$ and is equal to $\partial_{w}^{\prime}$.)

It follows from (1.11) and (1.12) that the endomorphisms $\partial_{w}$ and $\partial_{w}^{\prime}$ of $R(T)$ are $R(T)^{W}$-linear. Let $w_{0}$ be the longest Weyl group element. The most important property of Demazure's operators is the following formula, which relates the "top" operator $\partial_{w_{0}}$ to the Weyl character formula,

$$
\partial_{w_{0}}(u)=J(u) / \mathbf{d}
$$

for all $u \in R(T)$ ([10, Proposition 3]). Because of (1.2) this is equivalent to

$$
\partial_{w_{0}}=j^{*} j_{*} \text {. }
$$

Demazure has given a similar characterization of the operators $\partial_{w}$ for all $w \in W$; cf. Theorem 6.1.

\section{The Hecke algebra $\mathcal{D}$}

This section is in part a review of the work of Kazhdan and Lusztig [15] and Kostant and Kumar [17] on the representation ring $R(G)$ and the associated Hecke algebra $\mathcal{D}$. All results stated here follow readily from their work. As far as we are aware, the main novelty is the introduction of the augmentation left ideal of $\mathcal{D}$, which will play an important role in Section 4. The notation is as in Section 1. 
The augmentation left ideal. Let $\mathcal{E}=\operatorname{End}_{R(G)}(R(T))$ be the $R(G)$-algebra of $R(G)$-linear endomorphisms of $R(T)$. Let $\mathcal{D}$ be the subalgebra of $\mathcal{E}$ generated by the $\delta_{\alpha}$ and the elements of $R(T)$ (regarded as multiplication operators). It follows from (1.5) that $\mathcal{D}$ contains the group ring $\mathbf{Z}[W]$ (viewed as an algebra of endomorphisms of $R(T)$ ). It follows from Theorem 1.1 that $\partial_{w}, \partial_{w}^{\prime} \in \mathcal{D}$ for all $w$. We define the augmentation left ideal of $\mathcal{D}$ to be the annihilator of the identity element $1 \in R(T)$,

$$
I(\mathcal{D})=\{\Delta \in \mathcal{D} \mid \Delta(1)=0\} .
$$

Note that $I(\mathcal{D})$ contains the augmentation ideal $I(W)$ of $\mathbf{Z}[W]$ (whence its name), as well as the operators $\delta_{\alpha}^{\prime}$ for all $\alpha \in \mathcal{R}$ and $\partial_{w}^{\prime}$ for all $w \neq 1$.

The next result follows immediately from work of Kostant and Kumar. It says that $\mathcal{D}$ and $I(\mathcal{D})$ are free left $R(T)$-modules, and that as a ring $\mathcal{D}$ is isomorphic to the Hecke algebra (cf. $[15, \S 2.12]$ ) over $\mathbf{Z}$ of the extended affine Weyl group $\mathcal{X}(T) \rtimes W$.

\subsection{Theorem. $\quad$ (i) $\left(\partial_{w}\right)_{w \in W}$ is a basis of the left $R(T)$-module $\mathcal{D}$.}

(ii) $\left(\partial_{w}^{\prime}\right)_{w \in W}$ is a basis of the left $R(T)$-module $\mathcal{D}$.

(iii) $\left(\partial_{w}^{\prime}\right)_{w \neq 1}$ is a basis of the left $R(T)$-module $I(\mathcal{D})$.

(iv) Let $S$ be the set of simple reflections. The multiplication law of $\mathcal{D}$ is determined by that of $R(T)$ and by the rules

$$
\left[\partial_{s}^{\prime}, u\right]=\partial_{s}^{\prime}(u) s, \quad \partial_{s}^{\prime} \partial_{w}^{\prime}= \begin{cases}\partial_{s w}^{\prime} & \text { if } l(s w)=l(w)+1 \\ \partial_{w}^{\prime} & \text { if } l(s w)=l(w)-1\end{cases}
$$

for all $s \in S, w \in W$ and $u \in R(T)$.

Proof. Let $K$ be the fraction field of $R(T)$. Then $K^{W}$ is the fraction field of $R(T)^{W} \cong$ $R(G)$. Let $\mathcal{A}$ be the subalgebra of the twisted group algebra $K[W]$ which stabilizes $R(T)$. Then $\delta_{\alpha} \in \mathcal{A}$ for all $\alpha \in \mathcal{R}$, so $\mathcal{D}$ is a subalgebra of $\mathcal{A}$. By [17, Theorem 2.9] (where $K[W]$ is denoted by $Q_{W}$ and $\mathcal{A}$ by $Y$ ), $\mathcal{A}$ is a free left $R(T)$-module with basis $\left(\partial_{w}\right)_{w \in W}$. This shows that $\mathcal{A}=\mathcal{D}$, and hence (i). It follows from the Leibniz rule (1.8) and from (1.10) that the operators $\partial_{w}^{\prime}$ generate the same left $R(T)$-submodule of $\mathcal{E}$ as the operators $\partial_{w}$. Therefore $\left(\partial_{w}^{\prime}\right)_{w \in W}$ is also a basis of $\mathcal{D}$. By virtue of the fact that $\partial_{1}^{\prime}$ is the identity operator and that $\partial_{w}^{\prime}(1)=0$ for $w \neq 1$, this implies that $I(\mathcal{D})$ is the submodule spanned by the tuple $\left(\partial_{w}^{\prime}\right)_{w \neq 1}$. The commutation rule $\left[\partial_{s}^{\prime}, u\right]=\partial_{s}^{\prime}(u) s$ is equivalent to the product rule (1.11). That $\partial_{s}^{\prime} \partial_{w}^{\prime}=\partial_{s w}^{\prime}$ if $l(s w)=l(w)+1$ follows from the definition of the $\partial_{w}^{\prime}$. If $l(s w)=l(w)-1$, there is a reduced expression $w=s s_{1} \cdots s_{k}$ for $w$, so

$$
\partial_{s}^{\prime} \partial_{w}^{\prime}=\partial_{s}^{\prime} \partial_{s}^{\prime} \partial_{s_{1}}^{\prime} \cdots \partial_{s_{k}}^{\prime}=\partial_{s}^{\prime} \partial_{s_{1}}^{\prime} \cdots \partial_{s_{k}}^{\prime}=\partial_{w}^{\prime}
$$

by Theorem 1.1 and (1.10). Since $R(T)$ and the $\partial_{s}^{\prime}$ generate $\mathcal{D}$, these rules determine the algebra structure of $\mathcal{D}$.

(It follows from [17] that (i) and (ii) are true also for the right $R(T)$-module structure on $\mathcal{D}$. The left ideal $I(\mathcal{D})$, on the other hand, is not a right $R(T)$-module.)

The $R(G)$-bilinear form. Let $\mathcal{D}_{0}$ be the subalgebra of $\mathcal{E}=\operatorname{End}_{R(G)}(R(T))$ generated by the endomorphism $\partial_{w_{0}}$ and by the elements of $R(T)$. Then we have inclusions

$$
\mathcal{D}_{0} \subseteq \mathcal{D} \subseteq \mathcal{E}
$$


Now assume that $\pi_{1}(G)$ is torsion-free, i.e. that the derived subgroup of $G$ is simply connected. We claim that $\mathcal{D}_{0}=\mathcal{D}=\mathcal{E}$ in that case. The proof is based on the symmetric pairing $\mathcal{P}: R(T) \times R(T) \rightarrow R(G)$ defined by

$$
\mathcal{P}\left(u_{1}, u_{2}\right)=j_{*}\left(u_{1} u_{2}\right)
$$

for $a_{1}, a_{2} \in R(T)$, where $j_{*}$ is the holomorphic induction map of Section 1. It follows from (1.1) that this pairing is $R(G)$-bilinear. Pittie [23] and Steinberg [31] showed that the $R(G)$-module $R(T)$ is free of rank $|W|$. Kazhdan and Lusztig [15, Proposition 1.6] showed that the pairing $\mathcal{P}$ is nonsingular in the sense that the induced map

$$
\mathcal{P}^{\sharp}: R(T) \rightarrow R(T)^{\vee}
$$

is an isomorphism, where $R(T)^{\vee}=\operatorname{Hom}_{R(G)}(R(T), R(G))$ denotes the $R(G)$-dual of the module $R(T)$. (See also Panin [22, Theorem 8.1], who noted that this fact is implicit in an older result of Hulsurkar [14]; cf. also Merkurjev [20, Proposition 2.17].) We regard $R(T)^{\vee}$ as an $R(T)$-module with scalar multiplication given by $\left(u_{1} \cdot \phi\right)\left(u_{2}\right)=\phi\left(u_{1} u_{2}\right)$ for $u_{1}, u_{2} \in R(T)$ and $\phi \in R(T)^{\vee}$. Similarly, we view $\mathcal{E}$ as an $R(T) \otimes_{R(G)} R(T)$-module with scalar multiplication given by

$$
\left(u_{1} \otimes u_{2}\right) \cdot \Delta(u)=u_{1} \Delta\left(u_{2} u\right)
$$

for $u_{1}, u_{2}, u \in R(T)$ and $\Delta \in \mathcal{E}$.

2.2. Proposition. Assume that $\pi_{1}(G)$ is torsion-free.

(i) $R(T)^{\vee}$ is a free $R(T)$-module of rank 1 generated by the pushforward map $j_{*}$.

(ii) As an $R(G)$-algebra, $\mathcal{E}$ is isomorphic to the matrix algebra $\mathcal{M}_{|W|}(R(G))$. As an $R(T) \otimes_{R(G)} R(T)$-module, $\mathcal{E}$ is free of rank 1 on the generator $\partial_{w_{0}}$.

(iii) Let $\left(u_{w}\right)_{w \in W}$ be a basis of the $R(G)$-module $R(T)$. For every $\Delta \in \mathcal{E}$ there exist unique $b_{w, w^{\prime}} \in R(G)$ such that

$$
\Delta(u)=\sum_{w, w^{\prime} \in W} j^{*}\left(b_{w, w^{\prime}}\right) u_{w} \partial_{w_{0}}\left(u_{w^{\prime}} u\right)
$$

for all $u \in R(T)$.

(iv) We have $\mathcal{D}_{0}=\mathcal{D}=\mathcal{E}$.

Proof. Observe first that $j_{*} \in R(T)^{\vee}$ because $j_{*}$ is $R(G)$-linear. (i) now follows immediately from the fact that $\mathcal{P}^{\sharp}(1)=j_{*}$ and that $\mathcal{P}$ is nonsingular. The first observation in (ii) is obvious from the fact that $R(T)$ is free of rank $|W|$. Let $f$ be the composition of the natural $R(T) \otimes_{R(G)} R(T)$-linear maps

$$
R(T) \otimes_{R(G)} R(T) \stackrel{\mathcal{P}^{\sharp} \otimes 1}{\longrightarrow} R(T)^{\vee} \otimes_{R(G)} R(T) \stackrel{g}{\longrightarrow} \mathcal{E},
$$

where $g$ is defined by $g\left(\phi \otimes u_{1}\right)\left(u_{2}\right)=j^{*} \phi\left(u_{1} u_{2}\right)$. Then $g$ is an isomorphism because $R(T)$ is free and $\mathcal{P}^{\sharp} \otimes 1$ is an isomorphism because $\mathcal{P}$ is nonsingular. Moreover, $f(1 \otimes$ $1)=j^{*} j_{*}$, which is equal to $\partial_{w_{0}}$ by (1.13). This proves the second statement in (ii). The $|W|^{2}$-tuple $\left(u_{w} \otimes u_{w^{\prime}}\right)_{w, w^{\prime} \in W}$ is an $R(G)$-basis of $R(T) \otimes_{R(G)} R(T)$, so (iii) is a restatement of (ii). It follows from (iii) that $\mathcal{E} \subseteq \mathcal{D}_{0}$, which proves (iv).

For each character $\lambda \in \mathcal{X}(T)$, define $j_{\lambda}=e^{\lambda} \cdot j_{*} \in R(T)^{\vee}$, i.e. $j_{\lambda}(u)=j_{*}\left(e^{\lambda} u\right)$ for all $u \in R(T)$. We call $j_{\lambda}$ the twisted induction map with coefficients in the one-dimensional $T$-module defined by $\lambda$. 
2.3. Corollary. Assume that $\pi_{1}(G)$ is torsion-free. For each $\lambda \in \mathcal{X}(T)$, the twisted induction map $j_{\lambda}$ is a generator of the $R(T)$-module $R(T)^{\vee}$. The collection $\left(j_{\lambda}\right)_{\lambda \in \mathcal{X}(T)}$ is a basis of the abelian group $R(T)^{\vee}$.

Proof. The first assertion follows from Proposition 2.2(i) and the fact that $e^{\lambda}$ is a unit in $R(T)$. The second assertion follows from Proposition 2.2(i) and the fact that the $e^{\lambda}$ form a basis of $R(T)$.

Behaviour under covering maps. Let $\tilde{G}$ be a second compact connected Lie group and let $\phi: \tilde{G} \rightarrow G$ be a covering homomorphism. Let $\tilde{T}$ be the maximal torus $\phi^{-1}(T)$ of $\tilde{G}$. The pullback map $\phi^{*}: \mathcal{X}(T) \rightarrow \mathcal{X}(\tilde{T})$ is injective. It induces an injective homomorphism

$$
\phi^{*}: R(T) \rightarrow R(\tilde{T}),
$$

and it maps the root system of $G$ bijectively to that of $\tilde{G}$. We shall identify the two root systems via this bijection. For every root $\alpha$, the endomorphism $\delta_{\alpha}$ extends to an $R(\tilde{G})$-linear endomorphism $\tilde{\delta}_{\alpha}$ of $R(\tilde{T})$ given by the same formula as (1.5). Similarly, $\delta_{\alpha}^{\prime}$ extends to an $R(\tilde{G})$-linear endomorphism $\tilde{\delta}_{\alpha}^{\prime}$. Also, multiplication by an element $u \in R(T)$ extends in an obvious way to a multiplication operator on $R(\tilde{T})$. Thus we have defined an algebra homomorphism $\bar{\phi}: \mathcal{D} \rightarrow \tilde{\mathcal{D}}$, where $\tilde{\mathcal{D}}$ is the algebra of endomorphisms of $R(\tilde{T})$ generated by the $\tilde{\delta}_{\alpha}$ and by $R(\tilde{T})$. Observe that $\bar{\phi}$ maps $I(\mathcal{D})$ to $I(\tilde{\mathcal{D}})$.

2.4. Lemma. The homomorphism $\bar{\phi}: \mathcal{D} \rightarrow \tilde{\mathcal{D}}$ is injective and induces isomorphisms of left $R(\tilde{T})$-modules

$$
1 \otimes \bar{\phi}: R(\tilde{T}) \otimes_{R(T)} \mathcal{D} \rightarrow \tilde{\mathcal{D}}, \quad 1 \otimes \bar{\phi}: R(\tilde{T}) \otimes_{R(T)} I(\mathcal{D}) \rightarrow I(\tilde{\mathcal{D}}) .
$$

Proof. It follows from Theorem 1.1 that $\bar{\phi}\left(\partial_{w}\right)=\tilde{\partial}_{w}$ and $\bar{\phi}\left(\partial_{w}^{\prime}\right)=\tilde{\partial}_{w}^{\prime}$, where $\tilde{\partial}_{w}$ (resp. $\left.\tilde{\partial}_{w}^{\prime}\right)$ is the operator on $R(\tilde{T})$ analogous to $\partial_{w}$ (resp. $\partial_{w}^{\prime}$ ), so the statement follows from Theorem 2.1.

\section{3. $\mathcal{D}$-modules}

We shall see in Section 4 that the $T$-equivariant $\mathrm{K}$-group of a $G$-space is a module over the ring $\mathcal{D}$. In this section we collect some facts regarding abstract $\mathcal{D}$-modules. The notation is as in Sections 1 and 2.

Let $A$ be a left $\mathcal{D}$-module. We say an element of $A$ is $\mathcal{D}$-invariant or Hecke invariant if it is annihilated by all operators in the augmentation left ideal $I(\mathcal{D})$. We denote by $A^{I(\mathcal{D})}$ the group of invariants. By Theorem 2.1(iii),

$$
A^{I(\mathcal{D})}=\left\{a \in A \mid \partial_{w}^{\prime}(a)=0 \text { for all } w \neq 1\right\} .
$$

This group is not a $\mathcal{D}$-submodule of $A$, but a submodule over the ring $R(T)^{W} \cong$ $R(G)$. Since $I(\mathcal{D})$ contains the augmentation ideal $I(W)$ of $\mathbf{Z}[W]$, the Hecke invariants are contained in the Weyl invariants,

$$
A^{I(\mathcal{D})} \subseteq A^{W}
$$

an inclusion which is in general not an equality. On the other hand, for the $\mathcal{D}$-module $R(T)$ it follows from the definition (1.9) of the operators $\delta_{\alpha}^{\prime}$ that

$$
R(T)^{I(\mathcal{D})}=R(T)^{W} .
$$

This leads to the following characterization of the augmentation left ideal. 
3.1. Lemma. $I(\mathcal{D})=\left\{\Delta \in \mathcal{D} \mid \Delta \circ \partial_{w_{0}}=0\right\}$, the left annihilator of $\partial_{w_{0}}$.

Proof. Let $\Delta \in I(\mathcal{D})$ and $u \in R(T)$. It follows from (1.13) that $\partial_{w_{0}}(u)=j^{*} j_{*}(u)$ is in $R(T)^{W}=R(T)^{I(\mathcal{D})}$, so $\Delta \partial_{w_{0}}(u)=0$. Hence $\Delta \circ \partial_{w_{0}}=0$. Conversely, let $\Delta \in \mathcal{D}$ and suppose $\Delta \circ \partial_{w_{0}}=0$. It follows from (1.2) and (1.13) that $\partial_{w_{0}}(1)=1$. Therefore $\Delta(1)=\Delta \partial_{w_{0}}(1)=0$, and hence $\Delta \in I(\mathcal{D})$.

3.2. Lemma. Let $A$ be a $\mathcal{D}$-module. Then $\Delta(u a)=\Delta(u)$ a for all $\Delta \in \mathcal{D}, u \in R(T)$ and $a \in A^{I(\mathcal{D})}$.

Proof. The identity (1.12) holds if we replace $u_{1}$ with $u \in R(T)$ and $u_{2}$ with $a \in A$. Therefore, for each root $\alpha$, we have $\delta_{\alpha}(u a)=\delta_{\alpha}(u) a$ if $\delta_{\alpha}^{\prime}(a)=0$. Let $a \in A^{I(\mathcal{D})}$. Then $\delta_{\alpha}^{\prime}(a)=0$ for all $\alpha$, so $\delta_{\alpha}(u a)=\delta_{\alpha}(u) a$ for all $\alpha$, and hence $\partial_{w}(u a)=\partial_{w}(u) a$ for all $w \in W$ by Theorem 1.1. It now follows from Theorem 2.1(i) that $\Delta(u a)=\Delta(u) a$ for all $\Delta \in \mathcal{D}$.

The next two results give a measure of the discrepancy between $A^{W}$ and $A^{I(\mathcal{D})}$. In Theorem 4.7, Lemma 3.3(i) will be interpreted as a "Weyl character formula".

3.3. Lemma. Choose $u_{0} \in R(T)$ satisfying $\partial_{w_{0}}\left(u_{0}\right)=1$, e.g. $u_{0}=1$. Define $\pi \in \mathcal{D}$ by $\pi(u)=\partial_{w_{0}}\left(u_{0} u\right)$ for $u \in R(T)$. Let

$$
\mathcal{J}=\mathcal{D} \cdot \pi+\sum_{w \in W} \mathcal{D} \cdot(1-w)
$$

be the left ideal of $\mathcal{D}$ generated by $\pi$ and $I(W)$. Let $A$ be a left $\mathcal{D}$-module.

(i) $\pi: A \rightarrow A$ projects $A$ onto the $R(T)^{W}$-submodule $A^{I(\mathcal{D})}$.

(ii) $A^{W}=A^{I(\mathcal{D})} \oplus A^{\mathcal{J}}$. Hence $A^{W}=A^{I(\mathcal{D})}$ if and only if $A^{\mathcal{J}}=0$.

Proof. It follows from Lemma 3.1 that $\pi$ maps $A$ to $A^{I(\mathcal{D})}$. If $a \in A^{I(\mathcal{D})}$, then Lemma 3.2 shows that

$$
\pi(a)=\partial_{w_{0}}\left(u_{0} a\right)=\partial_{w_{0}}\left(u_{0}\right) a=a,
$$

since $\partial_{w_{0}}\left(u_{0}\right)=1$. This proves that $\pi(A)=A^{I(\mathcal{D})}$ and $\pi^{2}=\pi$, which establishes (i). It follows from (i) that $A$ is the direct sum of the $R(T)^{W}$-submodules $A^{I(\mathcal{D})}$ and $\operatorname{ker} \pi$. Moreover, it follows from (i) and (3.1) that $\pi$ maps $A^{W}$ into itself. Therefore $A^{W}$ is the direct sum of $A^{I(\mathcal{D})}$ and $A^{W} \cap \operatorname{ker} \pi=A^{\mathcal{J}}$.

3.4. Remark. Let $\mathbf{k}$ be an arbitrary commutative ring. For a $\mathbf{Z}$-module $M$, denote by $M_{\mathbf{k}}$ the $\mathbf{k}$-module $\mathbf{k} \otimes \mathbf{z} M$. Lemma 3.3 generalizes in an obvious way if we extend scalars from $\mathbf{Z}$ to $\mathbf{k}$. Thus, if we take $u_{0}$ to be any element of $R(T)_{\mathbf{k}}$ satisfying $\partial_{w_{0}}\left(u_{0}\right)=1$ and define $\pi \in \mathcal{D}_{\mathbf{k}}$ by $\pi(u)=\partial_{w_{0}}\left(u_{0} u\right)$ for $u \in R(T)_{\mathbf{k}}$, then $\pi$ projects $A$ onto $A^{I\left(\mathcal{D}_{\mathbf{k}}\right)}$ for any left $\mathcal{D}_{\mathbf{k}}$-module $A$.

Recall that $\mathbf{d}=\prod_{\alpha \in \mathcal{R}^{+}}\left(1-e^{-\alpha}\right) \in R(T)$ is the Weyl denominator (1.4). For simplicity we denote its image $1_{\mathbf{k}} \otimes \mathbf{d}$ in $R(T)_{\mathbf{k}}$ also by $\mathbf{d}$.

3.5. Lemma. The notation and the assumptions are as in Remark 3.4.

(i) We have $\mathbf{d} \in \mathcal{J}_{\mathbf{k}}$. Hence $A^{W}=A^{I\left(\mathcal{D}_{\mathbf{k}}\right)}$ if $\mathbf{d}$ is not a zero divisor in $A$.

(ii) Assume that $|W|$ is invertible in $\mathbf{k}$ and let $u_{0}=|W|^{-1} \mathbf{d} \in R(T)_{\mathbf{k}}$. Then $\partial_{w_{0}}\left(u_{0}\right)=1$ and $\pi: A \rightarrow A$ is the projection map onto the $W$-invariants,

$$
\pi=\frac{1}{|W|} \sum_{w \in W} w
$$


Hence $A^{W}=A^{I\left(\mathcal{D}_{\mathbf{k}}\right)}$.

Proof. The $\rho$-shifted Weyl action $w \odot u=e^{-\rho} w\left(e^{\rho} u\right)$ on $R(T)$ has the evident properties

$$
w \odot\left(u_{1} u_{2}\right)=\left(w \odot u_{1}\right) w\left(u_{2}\right), \quad w \odot J(u)=J(w \odot u)=\operatorname{det}(w) J(u)
$$

for all $w \in W$ and $u_{1}, u_{2}, u \in R(T)$, where $J$ is the $\rho$-shifted antisymmetrizer (1.3). It follows from (1.13) and (3.2) that for all $u \in R(T)$

$$
\begin{aligned}
\mathbf{d} \pi(u) & =\mathbf{d} \partial_{w_{0}}\left(u_{0} u\right)=J\left(u_{0} u\right)=J\left(u_{0}\right) u+J\left(u_{0} u\right)-J\left(u_{0}\right) u \\
& =\mathbf{d} \partial_{w_{0}}\left(u_{0}\right) u+\sum_{w} \operatorname{det}(w)\left(w \odot u_{0}\right)(w-1)(u) \\
& =\mathbf{d} u+\sum_{w} \operatorname{det}(w)\left(w \odot u_{0}\right)(w-1)(u) .
\end{aligned}
$$

Hence

$$
\mathbf{d}=\mathbf{d} \pi+\sum_{w} \operatorname{det}(w)\left(w \odot u_{0}\right)(1-w) \in \mathcal{J} .
$$

Therefore, if $\mathbf{d}$ is not a zero divisor in $A$, then $A^{\mathcal{J}}=0$, so $A^{W}=A^{I(\mathcal{D})}$ by Lemma 3.3(ii). The same argument holds if we extend scalars from $\mathbf{Z}$ to $\mathbf{k}$. This proves (i). Now assume that $|W|$ is invertible in $\mathbf{k}$. It follows from (3.2) that $w \odot \mathbf{d}=\operatorname{det}(w) \mathbf{d}$ for all $w \in W$. Hence, by (1.13) and (3.2),

$$
\begin{aligned}
\partial_{w_{0}}(\mathbf{d} u)=\frac{1}{\mathbf{d}} J(\mathbf{d} u)=\frac{1}{\mathbf{d}} \sum_{w} \operatorname{det}(w) w \odot(\mathbf{d} u) & \\
& =\frac{1}{\mathbf{d}} \sum_{w} \operatorname{det}(w)(w \odot \mathbf{d}) w(u)=\sum_{w} w(u)
\end{aligned}
$$

for all $u \in R(T)_{\mathbf{k}}$. In the first place, this identity shows that $\partial_{w_{0}}(\mathbf{d})=|W|$, and so $\partial_{w_{0}}\left(u_{0}\right)=1$. Secondly, it shows that

$$
\pi(u)=\partial_{w_{0}}\left(u_{0} u\right)=\frac{1}{|W|} \sum_{w \in W} w(u) .
$$

Hence $A^{W}=A^{I\left(\mathcal{D}_{\mathbf{k}}\right)}$ by virtue of Remark 3.4.

\section{Push-pull operators}

As in the previous section, $G$ denotes a compact connected Lie group with maximal torus $T$, root system $\mathcal{R}$ and Weyl group $W$. We fix a basis of $\mathcal{R}$ and define the operators $\partial_{w}$ and $\partial_{w}^{\prime}$ as in Theorem 1.1. We denote by $X$ a compact topological space on which $G$ acts continuously.

The algebra $\mathcal{D}$ acts on K-theory. As in [27] we denote by

$$
K_{G}^{*}(X)=K_{G}^{0}(X) \oplus K_{G}^{-1}(X)
$$

the equivariant K-theory of $X$. Recall that

$$
K_{G}^{*}(\mathrm{pt})=K_{G}^{0}(\mathrm{pt})=R(G)
$$

is the representation ring of $G$, where pt denotes a space consisting of a single point.

Let $j: T \rightarrow G$ be the inclusion map and

$$
j^{*}: K_{G}^{*}(X) \longrightarrow K_{T}^{*}(X)
$$


the restriction homomorphism. As shown by Atiyah $[2, \S 4]$, the functional $j_{*} \in R(T)^{\vee}$ generalizes to a pushforward homomorphism

$$
j_{*}: K_{T}^{*}(X) \longrightarrow K_{G}^{*}(X) .
$$

He proved that $j_{*}$ is $K_{G}^{*}(X)$-linear and satisfies $j_{*}(1)=1$, and deduced from this the following "splitting principle".

4.1. Proposition ([2, Proposition 4.9]). $j_{*}$ is a left inverse of $j^{*}$. Hence $j^{*}$ is injective and maps $K_{G}^{*}(X)$ onto a direct summand of $K_{T}^{*}(X)$.

4.2. Remark. The compactness assumption on $X$ is frequently a nuisance in practice. A map $j_{*}$ with the above properties can presumably be defined for any paracompact $G$-space $X$, provided that we replace the compactly supported K-theory of [27] with the K-theory of $[4, \S 4]$. If this is true, and if the Künneth formula of Theorem 4.4 below generalizes to this setting, then it is a straightforward exercise to extend the results of Sections 4 and 5 to paracompact spaces.

The purpose of this section is to prove Theorem 4.6 below, which describes the subgroup $j^{*}\left(K_{G}^{*}(X)\right)$ in terms of divided difference operators. This subgroup is contained in, but not necessarily equal to, $K_{T}^{*}(X)^{W}$, the Weyl invariants in $K_{T}^{*}(X)$. (See Example 4.8.)

First we show how to extend the Demazure operators to K-theory. Prompted by (1.13), we define the endomorphism $\delta_{X}$ of $K_{T}^{*}(X)$ by

$$
\delta_{X}=j^{*} j_{*} .
$$

Similarly, let $\alpha$ be a root, let $G_{\alpha}$ be the centralizer in $G$ of $\operatorname{ker} \alpha$, and let $j_{\alpha}: T \rightarrow G_{\alpha}$ be the inclusion. Define the endomorphism $\delta_{\alpha, X}$ of $K_{T}^{*}(X)$ by

$$
\delta_{\alpha, X}=j_{\alpha}^{*} j_{\alpha, *} .
$$

To define the induction map $j_{\alpha, *}$ we must choose a complex structure on $G_{\alpha} / T$. We do this by identifying $G_{\alpha} / T$ with the complex homogeneous space $\left(G_{\alpha}\right)_{\mathbf{C}} / B$, where $B$ is the Borel subgroup of $\left(G_{\alpha}\right)_{\mathbf{C}}$ generated by $T_{\mathbf{C}}$ and the root space $\mathfrak{g}_{\mathbf{C}}^{-\alpha}$.

4.3. Lemma. The operator $\delta_{X}$ is $K_{G}^{*}(X)$-linear: $\delta_{X}\left(j^{*}(b) a\right)=j^{*}(b) \delta_{X}(a)$ for all $a \in K_{T}^{*}(X)$ and $b \in K_{G}^{*}(X)$. Hence $\delta_{X}\left(j^{*}(b)\right)=j^{*}(b)$ and $\delta_{X}^{2}=\delta_{X}$.

Proof. Since $j_{*}$ is a homomorphism of $K_{G}^{*}(X)$-modules,

$$
\delta_{X}\left(j^{*}(b) a\right)=j^{*} j_{*}\left(j^{*}(b) a\right)=j^{*}\left(b j_{*}(a)\right)=j^{*}(b) \delta_{X}(a)
$$

for all $a \in K_{T}^{*}(X)$ and $b \in K_{G}^{*}(X)$. Therefore, since $j_{*}(1)=1$,

$$
\delta_{X}\left(j^{*}(b)\right)=j^{*}(b) \delta_{X}(1)=j^{*}(b)
$$

and $\delta_{X}^{2}(a)=\delta_{X}\left(j^{*} j_{*}(a)\right)=j^{*} j_{*}(a)=\delta_{X}(a)$.

The operators $\delta_{\alpha, X}$ have the same properties, as one sees by applying Lemma 4.3 to the group $G=G_{\alpha}$.

To check that the $\delta_{\alpha, X}$ generate an action of the algebra $\mathcal{D}$ on $K_{T}^{*}(X)$, we invoke a special case of the equivariant Künneth theorem, which was established by the combined efforts of Hodgkin [12], Snaith [28], McLeod [19], and Rosenberg and Schochet $[26]$. 
4.4. Theorem. Assume that $\pi_{1}(G)$ is torsion-free. Then the map

$$
R(T) \otimes_{R(G)} K_{G}^{*}(X) \rightarrow K_{T}^{*}(X)
$$

defined by $u \otimes b \mapsto u \cdot j^{*}(b)$ is an isomorphism of $\mathbf{Z} / 2 \mathbf{Z}$-graded $R(T)$-algebras.

Let $\mathcal{E}(X)$ be the ring of $K_{G}^{*}(X)$-linear endomorphisms of the abelian group $K_{T}^{*}(X)$. Let $R(T) \rightarrow \mathcal{E}(X)$ be the homomorphism defining the natural $R(T)$-module structure on $K_{T}^{*}(X)$. Let $\mathcal{S}$ be the set $\left\{\delta_{\alpha} \mid \alpha \in \mathcal{R}\right\}$ and define a map from $\mathcal{S}$ to $\mathcal{E}(X)$ by $\delta_{\alpha} \mapsto \delta_{\alpha, X}$. By definition, the set $\mathcal{S} \cup R(T)$ generates the ring $\mathcal{D}$, and the following statement says that the map $\mathcal{S} \cup R(T) \rightarrow \mathcal{E}(X)$ just defined extends uniquely to a ring homomorphism $\mathcal{D} \rightarrow \mathcal{E}(X)$.

4.5. Proposition. The operators $\delta_{\alpha, X}$, for $\alpha \in \mathcal{R}$, together with the natural $R(T)$ module structure define a unique $\mathcal{D}$-module structure on $K_{T}^{*}(X)$. This $\mathcal{D}$-module structure commutes with the $K_{G}^{*}(X)$-module structure and is contravariant with respect to $G$-equivariant continuous maps and covering homomorphisms of $G$.

Proof. First assume that $\pi_{1}(G)$ is torsion-free. Identify $K_{T}^{*}(X)$ with $R(T) \otimes_{R(G)} K_{G}^{*}(X)$ through the isomorphism of Theorem 4.4. Then

$$
\mathcal{E}(X)=\operatorname{End}_{K_{G}^{*}(X)}\left(K_{T}^{*}(X)\right)=\mathcal{E} \otimes_{R(G)} K_{G}^{*}(X),
$$

so the map $\mathcal{D} \rightarrow \mathcal{E}(X)$ defined by $\Delta \mapsto \Delta \otimes 1$, where 1 is the identity automorphism of $K_{G}^{*}(X)$, is a well-defined algebra homomorphism. It follows from (1.13) (applied to the group $G=G_{\alpha}$ ) and from Lemma 4.3 that $\delta_{\alpha, X}=\delta_{\alpha} \otimes 1$. This proves that the endomorphisms $\delta_{\alpha, X}$ generate a well-defined action of $\mathcal{D}$ on $K_{T}^{*}(X)$. If $\pi_{1}(G)$ is not torsion-free, we choose a covering $\phi: \tilde{G} \rightarrow G$ of $G$ by a compact connected $\tilde{G}$ such that $\pi_{1}(\tilde{G})$ is torsion-free. (For instance, we can take $\tilde{G}$ to be the direct product of a simply connected group and a torus.) According to a result of Snaith [28, Lemma 2.4], the pullback map

$$
\phi^{*}: K_{T}^{*}(X) \longrightarrow K_{\tilde{T}}^{*}(X)
$$

is injective, where $\tilde{T}$ is the maximal torus $\phi^{-1}(T)$ of $\tilde{G}$. Let $\tilde{\delta}_{\alpha, X}=\tilde{\jmath}_{\alpha}^{*} \tilde{\jmath}_{\alpha, *}$ be the operator on $K_{\tilde{T}}^{*}(X)$ corresponding to $\alpha$, where $\tilde{\jmath}_{\alpha}: \tilde{T} \rightarrow \tilde{G}_{\alpha}$ is the inclusion. It follows from the naturality properties of $j_{\alpha}^{*}$ and $j_{\alpha, *}$ that

$$
\phi^{*} \delta_{\alpha, X}=\tilde{\delta}_{\alpha, X} \phi^{*}
$$

Recall from Lemma 2.4 that $\phi$ induces an injective algebra homomorphism

$$
\bar{\phi}: \mathcal{D}=\bigoplus_{w} R(T) \partial_{w} \longrightarrow \tilde{\mathcal{D}}=\bigoplus_{w} R(\tilde{T}) \tilde{\partial}_{w}
$$

We already know that the $\tilde{\delta}_{\alpha, X}$ generate a well-defined $\tilde{\mathcal{D}}$-action on $K_{\tilde{T}}^{*}(X)$. The restriction of this action to the subalgebra $\mathcal{D}$ preserves the submodule $K_{T}^{*}(X)$ and, because of (4.3), the elements $\delta_{\alpha}$ act in the required fashion. The $\mathcal{D}$-module structure on $K_{T}^{*}(X)$ so defined is unique because the $\delta_{\alpha}$ and $R(T)$ generate the ring $\mathcal{D}$, and it commutes with the $K_{G}^{*}(X)$-module structure because of Lemma 4.3. The naturality properties with respect to equivariant maps and covering homomorphisms follow from the corresponding properties of $j^{*}$ and $j_{*}$. 
We will write the product (with respect to the module structure given by the proposition) of a class $a \in K_{T}^{*}(X)$ by an operator $\Delta \in \mathcal{D}$ as $\Delta_{X}(a)$, or as $\Delta(a)$ when there is no danger of ambiguity. The formulæ (1.5) and (1.9) translate into the identities

$$
s_{\alpha}=e^{\alpha}-\left(e^{\alpha}-1\right) \delta_{\alpha}=1-\left(1-e^{-\alpha}\right) \delta_{\alpha}^{\prime}
$$

of operators on $K_{T}^{*}(X)$.

Hecke invariants versus Weyl invariants. The next result means that a $T$-equivariant $\mathrm{K}$-class on $X$ is $G$-equivariant if and only if it is annihilated by the operators $\partial_{w}^{\prime}$ for all $w \neq 1$.

4.6. Theorem. The map $j^{*}$ is an isomorphism from $K_{G}^{*}(X)$ onto $K_{T}^{*}(X)^{I(\mathcal{D})}$.

Proof. First assume that $\pi_{1}(G)$ is torsion-free. Let $\mathfrak{A}=\mathcal{D}$ - $\mathfrak{M} \mathfrak{d} d$ and $\mathfrak{B}=R(G)$ $\mathfrak{M}$ od be the categories of (left) modules over the rings $\mathcal{D}$, resp. $R(G)$. By the PittieSteinberg theorem, the $R(G)$-module $R(T)$ is free, and therefore it is a progenerator of the category $\mathfrak{B}$. By Proposition 2.2(iv), $\mathcal{D}$ is the full endomorphism algebra of $R(T)$. Hence, by the first Morita equivalence theorem (see e.g. $[18, \S 18]$ ), the functor $\mathfrak{G}: \mathfrak{B} \rightarrow \mathfrak{A}$ defined by

$$
B \longmapsto R(T) \otimes_{R(G)} B
$$

is an equivalence with inverse $\mathfrak{F}: \mathfrak{A} \rightarrow \mathfrak{B}$ given by

$$
A \longmapsto \operatorname{Hom}_{\mathcal{D}}(R(T), A) .
$$

We assert that $\mathfrak{F}$ is naturally isomorphic to the functor $\mathfrak{I}: \mathfrak{A} \rightarrow \mathfrak{B}$ given by

$$
A \longmapsto A^{I(\mathcal{D})} \text {. }
$$

Indeed, define the natural $R(G)$-linear map $\Phi_{A}: \mathfrak{F}(A) \rightarrow A$ by $\Phi_{A}(f)=f(1)$. The map $\Phi_{A}$ is injective because $\mathcal{D} \supseteq R(T)$. By $\mathcal{D}$-linearity,

$$
\Delta\left(\Phi_{A}(f)\right)=\Delta(f(1))=f(\Delta(1))=0
$$

for all $f \in \mathfrak{F}(A)$ and $\Delta \in \mathcal{D}$, so $\Phi_{A}(f) \in \mathfrak{I}(A)$. For $a \in \mathfrak{I}(A)$ define $f_{a}: R(T) \rightarrow A$ by $f_{a}(u)=u a$. It follows from Lemma 3.2 that $f_{a} \in \mathfrak{F}(A)$, and clearly $\Phi_{A}\left(f_{a}\right)=$ a. Therefore the image of $\Phi_{A}$ is equal to $\mathfrak{I}(A)$. We conclude that $\Phi$ is a natural isomorphism from $\mathfrak{F}$ to $\mathfrak{I}$. Now consider the $\mathcal{D}$-module $A=K_{T}^{*}(X)$ and the $R(G)$ module $B=K_{G}^{*}(X)$. By Theorem 4.4, $A \cong \mathfrak{G}(B)$. Hence

$$
B \cong \mathfrak{F}(A) \cong \mathfrak{I}(A)=A^{I(\mathcal{D})} .
$$

If $\pi_{1}(G)$ is not torsion-free, we choose a covering group $\phi: \tilde{G} \rightarrow G$ as in the proof of Proposition 4.5 and consider the diagram (cf. $[19, \S 4]$ )

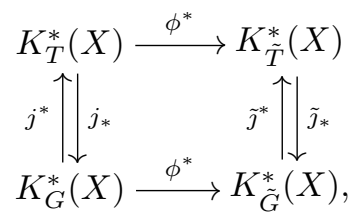

where we have $\phi^{*} \circ j^{*}=\tilde{\jmath}^{*} \circ \phi^{*}$ and $\tilde{\jmath}_{*} \circ \phi^{*}=\phi^{*} \circ j_{*}$. We know that $j_{*}$ is a left inverse of $j^{*}$ (Proposition 4.1), that the image of $\tilde{\jmath}^{*}$ is the submodule $\tilde{M}=K_{\tilde{T}}^{*}(X)^{I(\tilde{\mathcal{D}})}$ of $K_{\tilde{T}}^{*}(X)$ and that $\tilde{\jmath}_{*} \mid \tilde{M}$ is a two-sided inverse of $\tilde{\jmath}^{*}: K_{\tilde{G}}^{*}(X) \rightarrow \tilde{M}$. It follows from Lemma 2.4 that $\phi^{*}$ maps $M=K_{T}^{*}(X)^{I(\mathcal{D})}$ to $\tilde{M}$. As noted in the proof of Proposition 4.5, the 
$\operatorname{map} K_{T}^{*}(X) \rightarrow K_{\tilde{T}}^{*}(X)$ is injective, and hence so is the map $K_{G}^{*}(X) \rightarrow K_{\tilde{G}}^{*}(X)$. An easy diagram chase now shows that $j^{*}\left(K_{G}^{*}(X)\right)=M$.

An immediate consequence is the following statement, which for a space consisting of a single point amounts to the Weyl character formula.

4.7. Theorem. For every $a \in K_{T}^{*}(X)$ there exists a unique $b \in K_{G}^{*}(X)$ such that $j^{*}(b)=\partial_{w_{0}}(a)$.

Proof. It follows from (1.13) that $\partial_{w_{0}}$ acts on $K_{T}^{*}(X)$ as the operator $\delta_{X}$ defined in (4.1). Taking $A=K_{T}^{*}(X)$ and $u_{0}=1$ in Lemma 3.3(i), we see that $\partial_{w_{0}}$ projects $K_{T}^{*}(X)$ onto $K_{T}^{*}(X)^{I(\mathcal{D})}$. Now let $a \in K_{T}^{*}(X)$. Then, by Theorem $4.6, j_{*}(a)$ is the unique class $b \in K_{G}^{*}(X)$ satisfying $j^{*}(b)=\partial_{w_{0}}(a)$.

4.8. Example. This example, which generalizes [19, Remark 4.5], shows that in general $j^{*}$ is not an isomorphism onto $K_{T}^{*}(X)^{W}$, not even if $G$ is simply connected. Let $G=\mathbf{S U}(2)$ and let $X$ be any $G$-space. Let $T$ be the diagonal maximal torus of $G$ and let $\varpi \in \mathcal{X}(T)$ be the fundamental weight of $G$ defined by $\varpi\left(\begin{array}{ll}t & 0 \\ 0 & t^{-1}\end{array}\right)=t$. Then $\alpha=2 \varpi$ is the corresponding simple root, and

$$
R(T) \cong \mathbf{Z}\left[x, x^{-1}\right], \quad R(G) \cong \mathbf{Z}\left[x+x^{-1}\right],
$$

where $x=e^{\varpi}$. The elements 1 and $x$ are a basis of the $R(G)$-module $R(T)$. Relative to this basis the simple reflection $s_{\alpha}=w_{0}$ and the operator $\delta_{\alpha}^{\prime}=\partial_{w_{0}}^{\prime}$ are given by the matrices

$$
s_{\alpha}=\left(\begin{array}{cc}
1 & x+x^{-1} \\
0 & -1
\end{array}\right), \quad \delta_{\alpha}^{\prime}=\left(\begin{array}{ll}
0 & 0 \\
0 & 1
\end{array}\right) .
$$

By the Künneth theorem,

$$
K_{T}^{*}(X) \cong R(T) \otimes_{R(G)} K_{G}^{*}(X),
$$

so the classes $1 \otimes 1$ and $x \otimes 1$ form a basis of the $K_{G}^{*}(X)$-module $K_{T}^{*}(X)$, relative to which $s_{\alpha}$ and $\delta_{\alpha}^{\prime}$ act again as the matrices (4.4). Expressing an arbitrary class $a \in K_{T}^{*}(X)$ as $a=b_{1}+b_{2} x$ with $b_{1}, b_{2} \in K_{G}^{*}(X)$, we find by straightforward calculation

$$
\begin{gathered}
K_{T}^{*}(X)^{W}=\left\{b_{1}+b_{2} x \mid 2 b_{2}=\left(x+x^{-1}\right) b_{2}=0\right\}=K_{G}^{*}(X) \cdot 1 \oplus K_{G}^{*}(X)^{I} \cdot x, \\
K_{T}^{*}(X)^{I(\mathcal{D})}=\left\{b_{1}+b_{2} x \mid b_{2}=0\right\}=K_{G}^{*}(X) \cdot 1 .
\end{gathered}
$$

Here $I$ denotes the ideal of $R(G)$ generated by 2 and $x+x^{-1}$, and $K_{G}^{*}(X)^{I}$ denotes the $R(G)$-submodule of $K_{G}^{*}(X)$ annihilated by $I$. Thus $K_{T}^{*}(X)^{W}=j^{*}\left(K_{G}^{*}(X)\right)$ if and only if $K_{G}^{*}(X)$ contains no $I$-torsion elements. In particular, if $X$ is a principal $G$-bundle with base $Y=X / G$ such that $K^{*}(Y)$ has 2-torsion (e.g. $Y=\mathbf{P}^{n}(\mathbf{R})$ and $X=G \times Y$ ), then $K_{T}^{*}(X)^{W} \neq j^{*}\left(K_{G}^{*}(X)\right)$.

We close this section by stating some criteria for $K_{G}^{*}(X)$ to be isomorphic to $K_{T}^{*}(X)^{W}$.

4.9. Theorem. (i) Assume that the Weyl denominator $\mathbf{d}=\prod_{\alpha \in \mathcal{R}^{+}}\left(1-e^{-\alpha}\right)$ is not a zero divisor in the $R(T)$-module $K_{T}^{*}(X)$. Then $j^{*}$ is an isomorphism from $K_{G}^{*}(X)$ onto $K_{T}^{*}(X)^{W}$.

(ii) Let $\mathbf{k}$ be a commutative ring in which $|W|$ is invertible, such as $\mathbf{Z}\left[|W|^{-1}\right]$ or

Q. Then $j^{*}$ is an isomorphism from $K_{G}^{*}(X) \otimes \mathbf{k}$ onto $\left(K_{T}^{*}(X) \otimes \mathbf{k}\right)^{W}$.

Proof. This follows immediately from Theorem 4.6 and Lemma 3.5. 
4.10. Corollary. The map $j^{*}: K_{G}^{*}(X) \rightarrow K_{T}^{*}(X)^{W}$ is an isomorphism in each of the following cases.

(i) $K_{T}^{*}(X)$ is a torsion-free $R(T)$-module.

(ii) The restriction homomorphism $K_{T}^{*}(X) \rightarrow K_{T}^{*}\left(X^{T}\right)$ is injective.

(iii) $X$ is a compact Hamiltonian $G$-manifold.

(iv) $X$ is a nonsingular complex projective variety on which $G$ acts by linear transformations.

Proof. Case (i) follows immediately from Theorem 4.9(i). Let $A=K^{*}\left(X^{T}\right)$. Then the algebra $K_{T}^{*}\left(X^{T}\right)$ is isomorphic to the group algebra

$$
K_{T}^{*}\left(X^{T}\right) \cong A \otimes \mathbf{z} R(T) \cong A[\mathcal{X}(T)] .
$$

It follows from [6, $\S$ VI.3.2] that $1-e^{\alpha}$ is not a zero divisor in $A[\mathcal{X}(T)]$ for any root $\alpha$. Therefore case (ii) is also a consequence of Theorem 4.9(i). According to [11, Theorem 2.5], the assumption of case (ii) is satisfied in case (iii), so case (iii) follows from case (ii). Finally, case (iv) follows from case (iii), because a nonsingular subvariety of $\mathbf{P}^{n}(\mathbf{C})$ which is stable under the action of a subgroup $G$ of $\mathbf{U}(n+1)$ is a Hamiltonian $G$-manifold. (See e.g. $[16, \S 2]$.)

4.11. Example. Let $X$ be the flag variety $G / T$. Then $K_{G}^{*}(X) \cong K_{G \times T}^{*}(G) \cong R(T)$. To compute $K_{T}^{*}(X)$, let us assume that $\pi_{1}(G)$ is torsion-free. Then, by the Künneth theorem,

$$
K_{T}^{*}(X) \cong R(T) \otimes_{R(G)} K_{G}^{*}(X) \cong R(T) \otimes_{R(G)} R(T) \cong\left(R(T) \otimes_{\mathbf{Z}} R(T)\right) / I,
$$

where $I$ is the ideal of $R(T) \otimes_{\mathbf{z}} R(T)$ generated by all elements of the form $b \otimes 1-1 \otimes b$ with $b \in R(G)$. The isomorphism (4.5) is Weyl equivariant with respect to the $W$-action on $R(T) \otimes \mathbf{z} R(T)$ given by $w\left(a_{1} \otimes a_{2}\right)=w\left(a_{1}\right) \otimes a_{2}$. The inclusion $j^{*}: K_{G}^{*}(X) \rightarrow K_{T}^{*}(X)$ is induced by the map $R(T) \rightarrow R(T) \otimes \mathbf{z} R(T)$ which sends $a$ to $1 \otimes a$. We now conclude from (4.5) and the fact that $R(T)$ is a free $R(G)$-module that

$$
K_{T}^{*}(X)^{W} \cong\left(R(T) \otimes_{R(G)} R(T)\right)^{W} \cong R(T)^{W} \otimes_{R(G)} R(T) \cong R(T) \cong K_{G}^{*}(X) .
$$

Since the flag variety is complex projective, this follows also from Corollary 4.10(iv). The isomorphism (4.5) is $\mathcal{D}$-linear with respect to the $\mathcal{D}$-action on $R(T) \otimes \mathbf{z} R(T)$ given by $\Delta\left(a_{1} \otimes a_{2}\right)=\Delta\left(a_{1}\right) \otimes a_{2}$ (which preserves the ideal $\left.I\right)$. Therefore the projection onto the Weyl invariants

$$
\partial_{w_{0}}: R(T) \otimes_{R(G)} R(T) \longrightarrow R(T)
$$

is given by $\partial_{w_{0}}\left(a_{1} \otimes a_{2}\right)=\partial_{w_{0}}\left(a_{1}\right) a_{2}$.

4.12. Example. As a special case of Example 4.11, consider $G=\mathbf{S U}(2)$. Then $X=\mathbf{P}^{1}(\mathbf{C})$. Choose the maximal torus $T$ and the fundamental weight $\varpi$ as in Example 4.8. Then $R(T) \otimes_{\mathbf{Z}} R(T) \cong \mathbf{Z}\left[x, y,(x y)^{-1}\right]$, where $x$ and $y$ are two copies of the generator $e^{\varpi}$ of $R(T)$. The isomorphism (4.5) allows us to think of classes in $K_{T}^{*}(X)$ as cosets in $\mathbf{Z}\left[x, y,(x y)^{-1}\right]$ of the ideal $I$. Under this identification, the ring $K_{G}^{*}(X) \cong$ $K_{T}^{*}(X)^{W}$ corresponds to the subring $\mathbf{Z}\left[y, y^{-1}\right]$ of $\mathbf{Z}\left[x, y,(x y)^{-1}\right] / I$. By definition, $I$ is generated by all elements of the form $f(x)-f(y)$, where $f \in R(T)^{W}$ is a Weyl invariant Laurent polynomial in one variable. It follows that $I$ is generated by the single element 
$x+x^{-1}-\left(y+y^{-1}\right)$. Using (4.6) we can calculate the projection of the coset $x^{k} y^{l}+I$ onto $\mathbf{Z}\left[y, y^{-1}\right]$ for $k, l \in \mathbf{Z}$. We have $\partial_{w_{0}}=\delta_{\alpha}$, where $\alpha=2 \varpi$ is the simple root, so

$$
\partial_{w_{0}}\left(x^{k} y^{l}+I\right)=\delta_{\alpha}\left(y^{k}\right) y^{l}= \begin{cases}\left(y^{k}+y^{k-2}+\cdots+y^{-k}\right) y^{l} & \text { if } k \geq 0 \\ 0 & \text { if } k=-1 \\ -\left(y^{k+2}+y^{k+4}+\cdots+y^{-k-2}\right) y^{l} & \text { if } k \leq-2\end{cases}
$$

\section{Relative duality}

As in Section 4, $G$ denotes a compact connected Lie group with maximal torus $T$ and Weyl group $W$, and $X$ denotes a compact $G$-space. Choose a basis of the root system of $(G, T)$ and let $j_{*}: K_{T}^{*}(X) \rightarrow K_{G}^{*}(X)$ be the corresponding pushforward homomorphism. The pairing $\mathcal{P}$ defined in (2.1) generalizes to a bi-additive pairing $\mathcal{P}_{X}: K_{T}^{*}(X) \times K_{T}^{*}(X) \rightarrow K_{G}^{*}(X)$ defined by

$$
\mathcal{P}_{X}\left(a_{1}, a_{2}\right)=j_{*}\left(a_{1} a_{2}\right)
$$

for $a_{1}, a_{2} \in K_{T}^{*}(X)$. This pairing is graded symmetric in the sense that

$$
\mathcal{P}_{X}\left(a_{1}, a_{2}\right)=(-1)^{k_{1} k_{2}} \mathcal{P}_{X}\left(a_{2}, a_{1}\right)
$$

if $a_{1}$ is of degree $k_{1}$ and $a_{2}$ is of degree $k_{2}$. It is bilinear over $K_{G}^{*}(X)$ in the sense that

$$
\mathcal{P}_{X}\left(j^{*}(b) a_{1}, a_{2}\right)=b \mathcal{P}_{X}\left(a_{1}, a_{2}\right), \quad \mathcal{P}_{X}\left(a_{1}, a_{2} j^{*}(b)\right)=\mathcal{P}_{X}\left(a_{1}, a_{2}\right) b
$$

for all $b \in K_{G}^{*}(X)$. It follows from the naturality of $j_{*}$ that

$$
\mathcal{P}_{X}\left(f^{*}\left(a_{1}\right), f^{*}\left(a_{2}\right)\right)=f^{*} \mathcal{P}_{Y}\left(a_{1}, a_{2}\right)
$$

for $a_{1}, a_{2} \in K_{T}^{*}(Y)$, where $f: X \rightarrow Y$ is any $G$-equivariant continuous map.

Identifying $K_{T}^{*}(X)$ with $K_{G}^{*}(X \times G / T)$, we can view the pairing $\mathcal{P}_{X}$ as a fibrewise intersection product in $G$-equivariant K-theory for the projection map $X \times G / T \rightarrow X$, and the following proposition as a duality theorem for this map.

5.1. Proposition. Assume that $\pi_{1}(G)$ is torsion-free. Then the pairing $\mathcal{P}_{X}$ is nonsingular. Hence

$$
K_{T}^{*}(X) \cong \operatorname{Hom}_{K_{G}^{*}(X)}\left(K_{T}^{*}(X), K_{G}^{*}(X)\right)
$$

as $\mathbf{Z} / 2 \mathbf{Z}$-graded left $K_{G}^{*}(X)$-modules.

Proof. Let $\left(u_{w}\right)_{w \in W}$ be a basis of the $R(G)$-module $R(T)$ and $\left(u^{w}\right)_{w \in W}$ its $\mathcal{P}$-dual basis, which is characterized by $\mathcal{P}\left(u_{w}, u^{w^{\prime}}\right)=\delta_{w, w^{\prime}}$ for all $w, w^{\prime} \in W$. (As reviewed in Section 1, such bases exist because $\pi_{1}(G)$ is torsion-free.) Let $p: X \rightarrow$ pt be the constant map and define $\bar{u}_{w}=p^{*}\left(u_{w}\right)$ and $\bar{u}^{w}=p^{*}\left(u^{w}\right)$. By the Künneth theorem, Theorem 4.4, $\left(\bar{u}_{w}\right)_{w \in W}$ is a basis of the $K_{G}^{*}(X)$-module $K_{T}^{*}(X)$. It follows from (5.1) that

$$
\mathcal{P}_{X}\left(\bar{u}_{w}, \bar{u}^{w^{\prime}}\right)=p^{*} \mathcal{P}\left(u_{w}, u^{w^{\prime}}\right)=\delta_{w, w^{\prime}}
$$

for all $w, w^{\prime} \in W$. In other words, the tuple $\left(\bar{u}^{w}\right)_{w \in W}$ is a basis of $K_{T}^{*}(X)$ which is dual to $\left(\bar{u}_{w}\right)_{w \in W}$ with respect to the pairing $\mathcal{P}_{X}$.

5.2. Example. Assume that $\pi_{1}(G)$ is torsion-free. Taking $X=G$, equipped with the left multiplication action of $G$, we find from Proposition 5.1 that the pairing

$$
K^{*}(G / T) \times K^{*}(G / T) \rightarrow \mathbf{Z}
$$


is nonsingular, a form of Poincaré duality for the flag variety $G / T$. Taking $X=$ $G / T$, we find a $T$-equivariant Poincaré duality theorem for the flag variety, namely the assertion that the pairing

$$
K_{T}^{*}(G / T) \times K_{T}^{*}(G / T) \rightarrow R(T)
$$

is nonsingular. Taking $X=\mathrm{pt}$, we obtain $G$-equivariant Poincaré duality, namely the assertion that the pairing

$$
K_{G}^{*}(G / T) \times K_{G}^{*}(G / T) \rightarrow R(G)
$$

is nonsingular (which is equivalent to the pairing $\mathcal{P}$ on $R(T)$ being nonsingular).

\section{Algebraic equivariant K-theory}

The results of Sections 4 and 5 extend to the setting of algebraic K-theory thanks to the work of Thomason [32], Panin [22] and Merkurjev [20, 21]. As the arguments are closely parallel to those presented in the topological context, we will keep our exposition brief.

Let $\mathbf{k}$ be a field. By a variety we will mean a quasi-projective scheme over $\mathbf{k}$. All morphisms, sheaves, algebraic groups and their actions will be assumed to be defined over $\mathbf{k}$. We denote the point object $\operatorname{Spec}(\mathbf{k})$ by pt, and the unique morphism from a variety $X$ to pt by $p$ or $p_{X}$. We denote the group of characters (defined over $\mathbf{k}$ ) of an algebraic group $H$ by $\mathcal{X}(H)$.

Let $G$ be a split reductive group and $X$ a $G$-variety. We denote by $\mathfrak{M}^{G}(X)$ the category of $G$-equivariant coherent $\mathcal{O}_{X}$-modules, where $\mathcal{O}_{X}$ is the structure sheaf of $X$, and by $K_{*}^{G}(X)$ Quillen's K-theory of $\mathfrak{M}^{G}(X)$. Similarly, we denote by $\mathfrak{P}^{G}(X)$ the category of $G$-equivariant locally free coherent $\mathcal{O}_{X}$-modules and by $K_{G}^{*}(X)$ its K-theory. We have $\mathfrak{M}^{G}(\mathrm{pt})=\mathfrak{P}^{G}(\mathrm{pt})=\mathfrak{R e p}(G)$, the category of $G$-modules that are finitedimensional over $\mathbf{k}$, and $K_{0}^{G}(\mathrm{pt})=K_{G}^{0}(\mathrm{pt})=R(G)$, the Grothendieck ring of $\mathfrak{R e p}(G)$. The theory $K_{*}^{G}(X)$ is covariant with respect to projective $G$-morphisms, contravariant with respect to flat $G$-morphisms, and contravariant with respect to group homomorphisms. The theory $K_{G}^{*}(X)$ is contravariant with respect to arbitrary $G$-morphisms and with respect to group homomorphisms. Moreover, tensor multiplication induces a ring structure on $K_{G}^{0}(X)$ and a $K_{G}^{0}(X)$-module structure on $K_{*}^{G}(X)$.

Choose a Borel subgroup $B$ of $G$ and a split maximal torus $T$ of $B$. Let $j: T \rightarrow G$ and $k: T \rightarrow B$ denote the respective inclusions. These induce restriction maps

$$
j_{X}^{*}=j^{*}: K_{*}^{G}(X) \longrightarrow K_{*}^{T}(X), \quad k_{X}^{*}=k^{*}: K_{*}^{B}(X) \stackrel{\cong}{\longrightarrow} K_{*}^{T}(X),
$$

the second of which is an isomorphism by [20, Corollary 2.15]. The flag variety $\mathcal{B}=$ $G / B$ is projective, so the projection pr: $X \times \mathcal{B} \rightarrow X$ is a projective $G$-morphism. It is also flat and therefore induces homomorphisms

$$
\operatorname{pr}^{*}: K_{*}^{G}(X) \longrightarrow K_{*}^{G}(X \times \mathcal{B}), \quad \operatorname{pr}_{*}: K_{*}^{G}(X \times \mathcal{B}) \longrightarrow K_{*}^{G}(X) .
$$

The $B$-morphism $i: X \cong X \times\{B\} \rightarrow X \times \mathcal{B}$ induces an isomorphism

$$
i^{*}: K_{*}^{G}(X \times \mathcal{B}) \stackrel{\cong}{\longrightarrow} K_{*}^{B}(X)
$$

by [20, Proposition 2.10]. We have $j^{*}=k^{*} i^{*} \operatorname{pr}^{*}$ and we define a pushforward homomorphism by

$$
j_{X, *}=j_{*}=\operatorname{pr}_{*}\left(i^{*}\right)^{-1}\left(k^{*}\right)^{-1}: K_{*}^{T}(X) \longrightarrow K_{*}^{G}(X) .
$$


The fact that the variety $\mathcal{B}$ is smooth and rational implies that $j_{*}\left(\left[\mathcal{O}_{X}\right]\right)=\left[\mathcal{O}_{X}\right]$, where $\left[\mathcal{O}_{X}\right]$ is the class of the structure sheaf in $K_{0}^{T}(X)$, resp. $K_{0}^{G}(X)$. It now follows from the projection formula, $\left[24, \S 7\right.$, Proposition 2.10], that $j_{*}$ is a left inverse of $j^{*}$ and that $j^{*}\left(K_{*}^{G}(X)\right)$ is a direct summand of $K_{*}^{T}(X)$.

We now define the endomorphisms $\delta_{X}=j^{*} j_{*}$ and $\delta_{\alpha, X}=j_{\alpha}^{*} j_{\alpha, *}$ of $K_{*}^{T}(X)$ just as in Section 4. Showing that these operators generate a $\mathcal{D}$-action requires a number of auxiliary results.

Let $H$ be an algebraic group and let $Z$ be an $H \times B$-variety such that the quotient $Z \rightarrow Z / B$ exists and is a Zariski locally trivial principal $B$-bundle. Let $E_{\lambda}$ be the one-dimensional $B$-module defined by $\lambda \in \mathcal{X}(T) \cong \mathcal{X}(B)$, and let $\mathcal{L}_{\lambda} \in \mathfrak{M}^{H}(Z / B)$ be the sheaf of sections of the $H$-equivariant line bundle $Z \times{ }^{B} E_{\lambda}$ over $Z / B$. As in [9, $\S 1]$, we define the characteristic homomorphism

$$
c_{Z}^{H}=c^{H}: R(T) \longrightarrow K_{0}^{H}(Z / B)
$$

by $c^{H}\left(e^{\lambda}\right)=\left[\mathcal{L}_{\lambda}\right]$. Take $Z=\overline{B w B}$, the closure of the double coset of $w \in W$ in $G$, with $B$ acting by right multiplication, and take $H$ to be any closed subgroup of $G$ such that left multiplication by $H$ preserves $Z$. Then we obtain a homomorphism

$$
c_{w}^{H}: R(T) \longrightarrow K_{0}^{H}\left(S_{w}\right)
$$

where $S_{w}=\overline{B w B / B} \subseteq \mathcal{B}$ is the Schubert variety corresponding to $w$. (If $w=w_{0}$, then $S_{w}=\mathcal{B}$ and $H$ is allowed to be any closed subgroup of $G$.) The projective morphism $p_{w}=p_{S_{w}}: S_{w} \rightarrow$ pt induces a map

$$
p_{w, *}^{H}: K_{0}^{H}\left(S_{w}\right) \longrightarrow K_{0}^{H}(\mathrm{pt}) \cong R(H) .
$$

Taking $H=T$ we find an endomorphism $p_{w, *}^{T} \circ c_{w}^{T}$ of $R(T)$, which is equal to Demazure's operator $\partial_{w}$.

6.1. Theorem. We have $\partial_{w}=p_{w, *}^{T} \circ c_{w}^{T}$ for all $w \in W$, and $\partial_{w_{0}}=j_{\mathrm{pt}}^{*} \circ j_{\mathrm{pt}, *}$.

Proof. The first assertion is Demazure's character formula, $[9, \S 5$, Théorème 2], [1, Theorem 4.3]. Now let $w=w_{0}$, so that $S_{w}=\mathcal{B}$. Write $c_{w_{0}}^{H}=c^{H}$ for any closed subgroup $H$ of $G$ and $p_{w_{0}}=p$. It follows from the naturality properties of pullbacks and pushforwards $([24, \S 7.2])$ that the diagram

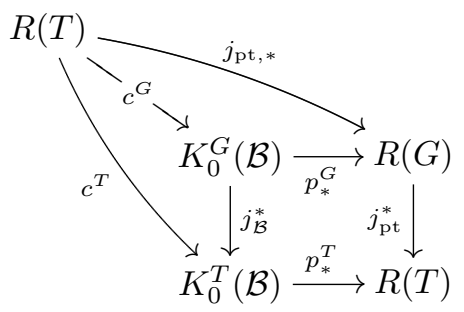

commutes, and hence that $\partial_{w_{0}}=p_{*}^{T} \circ c^{T}=j_{\mathrm{pt}}^{*} \circ j_{\mathrm{pt}, *}$.

The next statement is analogous to a result of Snaith [28, Lemma 2.4] and generalizes a result of Uma [33, Lemma 1.7].

6.2. Lemma. Let $\tilde{T}$ be a split torus and $\phi: \tilde{T} \rightarrow T$ an isogeny. Then, for every $T$-variety $X$, the map $R(\tilde{T}) \otimes_{R(T)} K_{*}^{T}(X) \rightarrow K_{*}^{\tilde{T}}(X)$ defined by $u \otimes a \mapsto u \cdot \phi^{*}(a)$ is an isomorphism. Hence the map $\phi^{*}: K_{*}^{T}(X) \rightarrow K_{*}^{\tilde{T}}(X)$ is injective and has an $R(T)$-linear left inverse. 
Proof. Let $\psi: C \rightarrow \tilde{T}$ be the reduced kernel of $\phi$ and let $\mathfrak{M}$ be the abelian category

$$
\mathfrak{M}=\prod_{\lambda \in \mathcal{X}(C)} \mathfrak{M}^{T}(X)
$$

We write an object of $\mathfrak{M}$ as an $\mathcal{X}(C)$-tuple $\left(\mathcal{F}_{\lambda}\right)_{\lambda \in \mathcal{X}(C)}$, where each $\mathcal{F}_{\lambda}$ is a $T$ equivariant coherent sheaf on $X$. The restriction homomorphism $\psi^{*}: \mathcal{X}(\tilde{T}) \rightarrow \mathcal{X}(C)$ is surjective $([30, \S 3.2])$; we choose a (set-theoretic) left inverse $\sigma$. Let $V_{\xi}$ be the onedimensional $\tilde{T}$-module defined by a character $\xi \in \mathcal{X}(\tilde{T})$. Let $\mathcal{G}$ be a $\tilde{T}$-equivariant coherent sheaf on $X$. Since $C$ acts trivially on $X$, the subsheaf $\mathcal{G}^{C}$ of $C$-invariant sections of $\mathcal{G}$ is a well-defined $T$-equivariant $\mathcal{O}_{X}$-module. The group $C$, being diagonalizable, is linearly reductive, so every locally finite $C$-module is a sum of isotypical components. (See e.g. $[29, \S$ III, $\S \mathrm{V}]$.) It follows that the subsheaf $\mathcal{G}^{C}$ is coherent. This enables us to define functors

$$
\mathfrak{M} \underset{\nu}{\stackrel{\mu}{\rightleftarrows}} \mathfrak{M}^{\tilde{T}}(X)
$$

by

$$
\begin{gathered}
\mu\left(\left(\mathcal{F}_{\lambda}\right)_{\lambda \in \mathcal{X}(C)}\right)=\bigoplus_{\lambda \in \mathcal{X}(C)} p^{*}\left(V_{\sigma(\lambda)}\right) \otimes_{\mathcal{O}_{X}} \phi^{*}\left(\mathcal{F}_{\lambda}\right), \\
\nu(\mathcal{G})=\left(\mathcal{H o m}_{\mathcal{O}_{X}}\left(p^{*}\left(V_{\sigma(\lambda)}\right), \mathcal{G}\right)^{C}\right)_{\lambda \in \mathcal{X}(C)} .
\end{gathered}
$$

Here $\phi^{*}(\mathcal{F})$ denotes a $T$-equivariant sheaf $\mathcal{F}$ regarded as a $\tilde{T}$-equivariant sheaf via the homomorphism $\phi$, and $\mathcal{H o m}_{\mathcal{O}_{X}}$ denotes sheaf hom. Then, for all objects $\left(\mathcal{F}_{\lambda}\right)_{\lambda \in \mathcal{X}(C)}$ of $\mathfrak{M}$,

$$
\begin{aligned}
\nu \mu\left(\left(\mathcal{F}_{\lambda}\right)_{\lambda}\right)=( & \left.\bigoplus_{\lambda} \mathcal{H o m}_{\mathcal{O}_{X}}\left(p^{*}\left(V_{\sigma\left(\lambda^{\prime}\right)}\right), p^{*}\left(V_{\sigma(\lambda)}\right) \otimes_{\mathcal{O}_{X}} \phi^{*}\left(\mathcal{F}_{\lambda}\right)\right)^{C}\right)_{\lambda^{\prime}} \\
& \cong\left(\bigoplus_{\lambda} \mathcal{H o m}_{\mathcal{O}_{X}}\left(p^{*}\left(V_{\sigma\left(\lambda^{\prime}\right)}\right), p^{*}\left(V_{\sigma(\lambda)}\right)\right)^{C} \otimes_{\mathcal{O}_{X}} \mathcal{F}_{\lambda}\right)_{\lambda^{\prime}} \\
& \cong\left(\bigoplus_{\lambda} p^{*}\left(\operatorname{Hom}_{\mathbf{k}}\left(V_{\sigma\left(\lambda^{\prime}\right)}, V_{\sigma(\lambda)}\right)^{C}\right) \otimes_{\mathcal{O}_{X}} \mathcal{F}_{\lambda}\right)_{\lambda^{\prime}} \cong\left(\mathcal{F}_{\lambda}\right)_{\lambda}
\end{aligned}
$$

and, for all objects $\mathcal{G}$ of $\mathfrak{M}^{\tilde{T}}(X)$,

$$
\mu \nu(\mathcal{G})=\bigoplus_{\lambda \in \mathcal{X}(C)} p^{*}\left(V_{\sigma(\lambda)}\right) \otimes_{\mathcal{O}_{X}} \phi^{*}\left(\mathcal{H o m}_{\mathcal{O}_{X}}\left(p^{*}\left(V_{\sigma(\lambda)}\right), \mathcal{G}\right)^{C}\right) \cong \mathcal{G}
$$

by the isotypical decomposition formula. We conclude that the abelian categories $\mathfrak{M}$ and $\mathfrak{M}^{\tilde{T}}(X)$ are equivalent and, by virtue of $[24, \S 2(8)]$, that the map

$$
\bigoplus_{\lambda \in \mathcal{X}(C)} K_{n}^{T}(X) \stackrel{\cong}{\longrightarrow} K_{n}^{\tilde{T}}(X)
$$

defined by $\left(a_{\lambda}\right)_{\lambda \in C} \mapsto \sum_{\lambda \in C} e^{\sigma(\lambda)} \cdot \phi^{*}\left(a_{\lambda}\right)$ is an isomorphism for all $n \in \mathbf{N}$. Setting $X=$ pt and $n=0$ in (6.1) gives $R(\tilde{T}) \cong \bigoplus_{\lambda \in \mathcal{X}(C)} R(T)$, and hence

$$
R(\tilde{T}) \otimes_{R(T)} K_{n}^{T}(X) \cong \bigoplus_{\lambda \in \mathcal{X}(C)} K_{n}^{T}(X)
$$


Combining the isomorphisms (6.1) and (6.2), we get

$$
R(\tilde{T}) \otimes_{R(T)} K_{n}^{T}(X) \cong K_{n}^{\tilde{T}}(X) .
$$

This isomorphism is induced by the functor $\mathfrak{R e p}(\tilde{T}) \times \mathfrak{M}^{T}(X) \rightarrow \mathfrak{M}^{\tilde{T}}(X)$ defined by $(E, \mathcal{F}) \mapsto p^{*}(E) \otimes_{\mathcal{O}_{X}} \phi^{*}(\mathcal{F})$, which proves the first assertion. Finally, the isomorphism (6.1) is $R(T)$-linear and the projection of $K_{n}^{\tilde{T}}(X)$ onto the direct summand corresponding to $\lambda=0$ is a left inverse of $\phi^{*}$.

A further ingredient we need is a Künneth formula of Merkurjev.

6.3. Theorem ([20, Proposition 4.1]). Assume that $\pi_{1}(G)$ is torsion-free. For every $G$-variety $X$, the map

$$
R(T) \otimes_{R(G)} K_{*}^{G}(X) \rightarrow K_{*}^{T}(X)
$$

defined by $u \otimes b \mapsto u \cdot j^{*}(b)$ is an isomorphism.

A point where the present treatment diverges from that of Section 4 is the absence of a ring structure on the group $K_{*}^{G}(X)$. Because of this there is no natural sense in which the $\delta_{\alpha, X}$ are $K_{*}^{G}(X)$-linear. Instead we have the following statement.

6.4. Lemma. Assume that $\pi_{1}(G)$ is torsion-free. Identify the $R(T)$-modules $K_{*}^{T}(X)$ and $R(T) \otimes_{R(G)} K_{*}^{G}(X)$ via the Künneth isomorphism. Then $\delta_{X}=\partial_{w_{0}} \otimes 1$, where 1 denotes the identity map of $K_{*}^{G}(X)$.

Proof. For all $u \in R(T)$ and $b \in K_{*}^{G}(X)$ we have

$$
\delta_{X}\left(u \cdot j^{*}(b)\right)=j^{*} j_{*}\left(u \cdot j^{*}(b)\right)=j^{*}\left(j_{*}(u) \cdot b\right)=j^{*} j_{*}(u) \cdot j^{*}(b)=\partial_{w_{0}}(u) \cdot j^{*}(b),
$$

where we used the projection formula and the naturality of $j^{*}$.

Under the same assumptions we have $\delta_{\alpha, X}=\delta_{\alpha} \otimes 1$ for all roots $\alpha$. With Theorem 6.1, Lemma 6.2, Theorem 6.3 and Lemma 6.4 in hand, one proves the next assertion in exactly the same way as Proposition 4.5.

6.5. Proposition. The operators $\delta_{\alpha, X}$, for $\alpha \in \mathcal{R}$, together with the natural $R(T)$ module structure generate a unique $\mathcal{D}$-module structure on $K_{T}^{*}(X)$. This $\mathcal{D}$-module structure is contravariant with respect to $G$-morphisms of varieties and isogenies of $G$.

The proof of the following result is now identical to that of Theorem 4.6.

6.6. Theorem. For every $G$-variety $X$, the map $j^{*}$ is an isomorphism from $K_{*}^{G}(X)$ onto $K_{*}^{T}(X)^{I(\mathcal{D})}$.

From this one easily deduces obvious analogues of Theorems 4.7 and 4.9 and Corollary $4.10(\mathrm{i})-(\mathrm{ii})$, which we leave it to the reader to state. The analogue of Corollary $4.10(\mathrm{iv})$ is as follows.

6.7. Corollary. Assume that $\mathbf{k}$ is perfect. Let $X$ be a smooth projective G-variety over k. Then $j^{*}: K_{*}^{G}(X) \rightarrow K_{*}^{T}(X)^{W}$ is an isomorphism.

Proof. It follows from [35, Theorem 2] that the restriction map $K_{*}^{T}(X) \rightarrow K_{*}^{T}\left(X^{T}\right)$ is injective. Now apply (the algebraic K-theory analogue of) Corollary 4.10(ii).

For a nonsingular variety the theories $K_{G}^{*}(X)$ and $K_{*}^{G}(X)$ are isomorphic and $K_{*}^{G}(X)$ is a graded commutative ring. (See e.g. [34, appendix].) The result of Section 5 therefore extends to algebraic K-theory in the following way. 
6.8. Proposition. Assume that $\pi_{1}(G)$ is torsion-free. Let $X$ be a smooth quasiprojective $G$-variety over $\mathbf{k}$. Then the pairing

$$
\mathcal{P}_{X}: K_{*}^{T}(X) \times K_{*}^{T}(X) \rightarrow K_{*}^{G}(X)
$$

defined by $\mathcal{P}_{X}\left(a_{1}, a_{2}\right)=j_{*}\left(a_{1} a_{2}\right)$ is nonsingular. Hence

$$
K_{*}^{T}(X) \cong \operatorname{Hom}_{K_{*}^{G}(X)}\left(K_{*}^{T}(X), K_{*}^{G}(X)\right)
$$

as $\mathbf{Z}$-graded left $K_{*}^{G}(X)$-modules.

\section{Acknowledgements}

We thank Samuel Evens and the referee for useful suggestions.

\section{References}

[1] H. Andersen, Schubert varieties and Demazure's character formula, Invent. Math. 79 (1985), no. 3, 611-618.

[2] M. Atiyah, Bott periodicity and the index of elliptic operators, Quart. J. Math. Oxford Ser. (2) 19 (1968), 113-140.

[3] M. Atiyah and R. Bott, A Lefschetz fixed point formula for elliptic complexes. I, Ann. of Math. (2) 86 (1967), 374-407, II. Applications, ibid. 88 (1968), 451-491.

[4] M. Atiyah and G. Segal, Equivariant K-theory and completion, J. Differential Geometry 3 (1969), $1-18$.

[5] R. Bott, The index theorem for homogeneous differential operators, Differential and Combinatorial Topology, A Symposium in Honor of Marston Morse (Princeton, NJ) (S. Cairns, ed.), Princeton Mathematical Series, vol. 27, Princeton University Press, 1965, pp. 167-186.

[6] N. Bourbaki, Groupes et algèbres de Lie, Éléments de mathématique, Diffusion CCLS, Paris, 1971, 1972, Masson, Paris, 1981, 1982, 1990.

[7] P. Bressler and S. Evens, The Schubert calculus, braid relations, and generalized cohomology, Trans. Amer. Math. Soc. 317 (1990), no. 2, 799-811.

[8] M. Demazure, Invariants symétriques entiers des groupes de Weyl et torsion, Invent. Math. 21 (1973), 287-301.

[9] , Désingularisation des variétés de Schubert généralisées, Ann. Sci. École Norm. Sup. (4) 7 (1974), 53-88.

[10] __ Une nouvelle formule des caractères, Bull. Sci. Math. (2) 98 (1974), no. 3, 163-172.

[11] M. Harada and G. Landweber, The K-theory of abelian symplectic quotients, Math. Res. Lett. 15 (2008), no. 1, 57-72.

[12] L. Hodgkin, The equivariant Künneth theorem in K-theory, Topics in K-theory, Lecture Notes in Mathematics, vol. 496, Springer-Verlag, Berlin, 1975, pp. 1-101.

[13] T. Holm and R. Sjamaar, Torsion and abelianization in equivariant cohomology, Transform. Groups 13 (2008), no. 3-4, 585-615.

[14] S. Hulsurkar, Proof of Verma's conjecture on Weyl's dimension polynomial, Invent. Math. 27 (1974), 45-52.

[15] D. Kazhdan and G. Lusztig, Proof of the Deligne-Langlands conjecture for Hecke algebras, Invent. Math. 87 (1987), no. 1, 153-215.

[16] F. Kirwan, Cohomology of quotients in symplectic and algebraic geometry, Mathematical Notes, vol. 31, Princeton University Press, Princeton, NJ, 1984.

[17] B. Kostant and S. Kumar, T-equivariant $K$-theory of generalized flag varieties, J. Differential Geom. 32 (1990), no. 2, 549-603.

[18] T. Y. Lam, Lectures on modules and rings, Graduate Texts in Mathematics, vol. 189, SpringerVerlag, New York, 1999.

[19] J. McLeod, The Künneth formula in equivariant K-theory, Algebraic Topology (Waterloo, 1978) (P. Hoffman and V. Snaith, eds.), Lecture Notes in Mathematics, vol. 741, Springer-Verlag, Berlin, 1979, pp. 316-333.

[20] A. Merkurjev, Comparison of equivariant and ordinary K-theory of algebraic varieties, St. Petersburg Math. J. 9 (1998), 815-850. 
[21] Equivariant $K$-theory, Handbook of $K$-theory (E. Friedlander and D. Grayson, eds.), vol. 2, Springer-Verlag, Berlin, 2005, pp. 925-954.

[22] I. Panin, On the algebraic K-theory of twisted flag varieties, $K$-Theory 8 (1994), no. 6, 541-585.

[23] H. Pittie, Homogeneous vector bundles on homogeneous spaces, Topology 11 (1972), 199-203.

$[24]$ D. Quillen, Higher algebraic $K$-theory I, Algebraic $K$-theory I: Higher $K$-theories (Battelle Memorial Inst., Seattle, WA, 1972) (H. Bass, ed.), Lecture Notes in Mathemathics, vol. 341, SpringerVerlag, Berlin, 1973, pp. 85-147.

[25] S. Ramanan and A. Ramanathan, Projective normality of flag varieties and Schubert varieties, Invent. Math. 79 (1985), no. 2, 217-224.

[26] J. Rosenberg and C. Schochet, The Künneth theorem and the universal coefficient theorem for equivariant K-theory and KK-theory, Mem. Amer. Math. Soc. 62 (1986), no. 348, vi+95.

[27] G. Segal, Equivariant K-theory, Inst. Hautes Études Sci. Publ. Math. 34 (1968), 129-151.

[28] V. Snaith, On the Künneth formula spectral sequence in equivariant K-theory, Proc. Cambridge Philos. Soc. 72 (1972), 167-177.

[29] T. Springer, Aktionen reduktiver Gruppen auf Varietäten, Algebraische Transformationsgruppen und Invariantentheorie (H. Kraft, P. Slodowy, and T. Springer, eds.), DMV Seminar, vol. 13, Birkhäuser, Basel, 1989, pp. 3-39.

[30] Linear algebraic groups, second ed., Progress in Mathematics, vol. 9, Birkhäuser, Boston, 1998.

[31] R. Steinberg, On a theorem of Pittie, Topology 14 (1975), 173-177.

[32] R. Thomason, Algebraic K-theory of group scheme actions, Algebraic topology and algebraic $K$ theory (Princeton, NJ, 1983) (W. Browder, ed.), Ann. of Math. Stud., vol. 113, Princeton Univ. Press, 1987, pp. 539-563.

[33] V. Uma, Equivariant K-theory of compactifications of algebraic groups, Transform. Groups 12 (2007), no. 2, 371-406.

[34] G. Vezzosi and A. Vistoli, Higher algebraic K-theory of group actions with finite stabilizers, Duke Math. J. 113 (2002), no. 1, 1-55.

[35] _ Higher algebraic K-theory for actions of diagonalizable groups, Invent. Math. 153 (2003), no. 1, 1-44, Erratum, ibid. 161 (2005), no. 1, 219-224.

Department of Mathematics and Statistics, McMaster University, Hamilton, ON L8S 4K1, CANADA

E-mail address: Megumi.Harada@math.mcmaster.ca

Department of Mathematics, Bard College, Annandale-on-Hudson, NY 12504-5000, USA

E-mail address: gregland@bard.edu

Department of Mathematics, Cornell University, Ithaca, NY 14853-4201, USA

E-mail address: sjamaar@math.cornell.edu 